FORMATION Formation emploi

Revue française de sciences sociales

121 | janvier-mars 2013

L'emploi public : nouveau système de règles, nouvelles stratégies d'acteurs

\title{
Entre travail politique et action administrative : les directions générales des services en régions
}

Between Political Work and Administrative Action: The executive directors of the French Regional Authorities

Zwischen politischer Arbeit und administrativer Handlung: die Directions générales des services (Generaldirektionen der Ämter von

Gebietskörperschaften) in den Regionen

Entre trabajo político y acción administrativa : las direcciones generales de servicios en las regiones

Sébastien Gardon et Eric Verdier

\section{(2) OpenEdition}

Journals

Édition électronique

URL : http://journals.openedition.org/formationemploi/3933

DOI : 10.4000/formationemploi.3933

ISSN : 2107-0946

Éditeur

La Documentation française

Édition imprimée

Date de publication : 15 février 2013

Pagination : 61-82

ISSN : 0759-6340

Référence électronique

Sébastien Gardon et Eric Verdier, «Entre travail politique et action administrative : les directions

générales des services en régions », Formation emploi [En ligne], 121 | janvier-mars 2013, mis en ligne le 15 février 2015, consulté le 30 octobre 2020. URL : http://journals.openedition.org/formationemploi/ 3933 ; DOI : https://doi.org/10.4000/formationemploi.3933

(c) Tous droits réservés 


\title{
Entre travail politique et action administrative : les directions générales des services en régions
}

\author{
SÉBASTIEN GARDON \\ Docteur en science politique et post-doctorant au Laboratoire Biens Normes Contrats (EA 3788, \\ université d'Avignon et des Pays de Vaucluse) \\ ERIC VERDIER \\ Sociologue et économiste \\ Directeur de recherche au CNRS - LEST (Laboratoire d'économie et de sociologie du travail \\ UMR 7317, CNRS et Aix-Marseille université)
}

Résumé

Entre travail politique et action administrative : les directions générales des services en régions

Cet article analyse les modes de gouvernance, les configurations organisationnelles et les modalités d'accès à la haute fonction publique régionale. Il revient sur la porosité des frontières entre activité politique et pilotage administratif et témoigne de l'instabilité des positions respectives des parties prenantes. Deux figures types de marchés des places de direction très opposés sont identifiées : un marché centré sur une régulation interne, l'autre sur le recours aux compétences externes, en priorité auprès des agents du partenaire clé des Régions que reste l'État. Dans un cas, cette situation renforce la dépendance et la proximité des fonctionnaires territoriaux avec les élus. Dans l'autre cas, elle conduit à la reconnaissance de la légitimité et de l'autonomie professionnelle des cadres de la fonction publique territoriale.

Mots clés : Fonction publique territoriale, cadre, recrutement, cheminement professionnel

Abstract

Between Political Work and Administrative Action: The executive directors of the French Regional Authorities

This paper analyzes the governance, the organization and the modes of access to the heads of administrative services in the regions. It shows that the boundaries between politics and administrative control are porous and the positions of different stakeholders unstable. Two very opposite types of job markets are identified: the first one is focused on internal 
management of places, the second ones relies primarily on external expertise brought by civil servants of the State, the privileged partner of the Regions. In one case, this situation reinforces dependency and proximity of regional executive directors with local elected officials. In either case, it leads to the recognition of the legitimacy and autonomy of the executive directors.

Key words: Regional civil service, managerial employee, recruitment, occupational paths Journal of Economic Literature: H 83, M 51

Traduction : Auteurs

Parmi les trois fonctions publiques, la fonction publique territoriale est celle qui a connu les plus fortes évolutions depuis ces vingt dernières années (Biland, 2009). Depuis l'Acte II de la décentralisation et les derniers transferts de personnels, elle compte près de deux millions d'agents (Biland, 2012). Les structures intercommunales ont sans doute connu les plus grandes transformations; cependant, les régions ont vécu un glissement de leur position d'administration de mission, pivot de projets territoriaux (Catlla, 2007), vers celui d'administration de gestion, que ce soit au niveau : du transfert de missions de plus en plus techniques avec de surcroît des périmètres d'intervention régulièrement reconfigurés (cas emblématique de la formation professionnelle, Bel et alii, 2003) ; de leurs ressources, avec une attrition croissante de leurs capacités de financement qui perturbe leur désormais classique travail de courtage ${ }^{1}$ (Nay, Smith, 2002) ; de leurs personnels, sachant que l'arrivée brutale des personnels techniciens, ouvriers et de service (TOS) de l'Éducation nationale a quadruplé leurs effectifs.

Dans un tel contexte d'incertitude sur les ressources, les compétences et les horizons d'actions de la collectivité dont ils ont la responsabilité, les membres des exécutifs régionaux construisent leurs activités de travail dans des configurations institutionnelles et organisationnelles encore peu stabilisées (Nay, 2002). Y contribue l'intervention récurrente d'un "État surplombant " (Salais, 1998) qui mobilise périodiquement le " coup d'État ${ }^{2}$ " comme technique de gouvernement (Padioleau, 1994), comme c'est le cas dans le domaine clé, pour les régions, de la formation professionnelle. Dans le cadre de cette institutionnalisation régionale encore en train de se faire, la division du

1. Il s’agit pour un élu régional d'obtenir des financements publics pour des projets localisés dans son territoire politique originel privilégié (commune ou structure intercommunale, canton ou encore département). 2. Périodiquement, l'État intervient dans des domaines ou selon des modalités qui interferent avec les champs de compétence des collectivités territoriales ou des partenaires sociaux sans concertation préalable avec les unes et les autres : citons, en 2011, la ponction du budget de l'État dans le fonds paritaire de sécurisation des parcours professionnels ou, antérieurement, la création des contrats première embauche et nouvelle embauche (CPE et CNE). 
travail entre politiques et techniciens s'avère être en recomposition constante, témoignant de la porosité des frontières entre les positions des uns et des autres (Le Lidec, 2012).

Précisément, cet article se focalise sur une composante majeure du travail politique régional, à savoir la direction générale des services (DGS). En effet, l'organisation des conseils régionaux s'est progressivement structurée autour de cette DGS ; son champ d'intervention a largement débordé du cadre administratif pour embrasser, dans le cours d'interactions complexes avec les élus de l'exécutif et le cabinet du président de la région, des tâches explicitement politiques telles que des propositions d'orientation pour l'action publique régionale. L'affirmation de la place de cette direction générale, fortement étoffée depuis la fin des années 90, ouvre cet article. Sa seconde partie démontre que le contenu précis des fonctions et la régulation de l'accès à ces dernières sont assez étroitement liés au mode de gouvernance de chaque région étudiée 3 .

\section{Encadré 1}

\section{Les différentes parties prenantes de la haute hiérarchie de la région}

Plusieurs pôles contribuent au fonctionnement des conseils régionaux. Les décisions sont prises par le président et son exécutif composé des vice-présidents et conseillers régionaux délégués et spéciaux. Le président est entouré d'un cabinet (composé d'une vingtaine de membres : directeur, directeur adjoint et chef de cabinet ainsi que conseillers techniques) qui assure la régulation politique du conseil régional ; selon des modalités variables, les vice-présidents bénéficient du concours d'un ou plusieurs collaborateurs. Le travail délibératif de préparation des politiques régionales et de discussion des rapports présentés en assemblée plénière (ou en commission permanente) est réalisé par différentes commissions thématiques de travail composées d'élus de la majorité et de l'opposition. Cette activité s'articule étroitement à l'action des différents groupes partisans de la majorité. Enfin la mise en œuvre des politiques régionales relève des services de la région avec, à leur tête, un directeur général des services et des directeurs généraux adjoints qui se chargent de la traduction opérationnelle des orientations politiques arrêtées par les élus. En réalité, comme le montre la première partie de cet article, cette distinction formelle entre des élus qui élaborent et des administratifs qui mettent en œuvre ne résiste pas complètement, loin s'en faut, à l'examen des pratiques.

3. Les matériaux sont issus d'une recherche qui porte sur cinq régions françaises d'assez grande taille. Toutes comptent plus de deux millions d'habitants, seuil retenu par la législation pour déterminer les carrières indiciaires des membres de la direction générale. La restitution de ces investigations s'attache à respecter le strict engagement de confidentialité et d'anonymat souscrit auprès de nos interlocuteurs. Sur le détail des sources, voir l'encadré méthodologique. 


\section{Encadré 2}

\section{Méthodologie}

Cet article repose en premier lieu sur l'exploitation d'une dizaine d'entretiens avec sept DGS (directeurs généraux des services) de quatre conseils régionaux (tous des hommes, de 46 à 58 ans, certains ayant été vus à plusieurs reprises) et d'une vingtaine d'entretiens avec quinze DGA (directeurs généraux adjoints) des mêmes conseils régionaux (six femmes et neuf hommes, de 40 à 60 ans certain(e)s ayant été interrogés plusieurs fois). Ces directeurs ont été choisis en fonction de leur position dans les organigrammes et des délégations d'élus de l'exécutif régional avec lesquelles ils étaient en interaction. D'une durée de une à quatre heures (en moyenne deux heures), ces entretiens semi-directifs ont été menés par un ou deux chercheurs dans les bureaux des acteurs rencontrés.

En termes de contenu, les entretiens étaient organisés en deux temps, de même durée : d'une part, les parcours biographiques des acteurs ; d'autre part, leurs activités au sein de la région. Deux séances de restitution en présence du DGS et de plusieurs DGA ont été organisées dans deux des régions étudiées en septembre 2010 et mars 2011.

En outre, de nombreux autres entretiens ont été recoupés avec les précédents pour contribuer à l'analyse des pratiques de recrutement, des logiques de mobilité interne et externe propres aux cadres dirigeants des régions étudiées, ainsi que de la répartition des responsabilités et des activités entre ces derniers, les élus de l'exécutif et le cabinet du président de la région : ainsi ont été interrogés dix-sept vice-présidents (dont certains plusieurs fois), leurs collaborateurs directs, des présidents de commission, des conseillers régionaux délégués, des présidents de groupe, des collaborateurs de groupe, des responsables de services (directeurs, délégués généraux à un thème particulier, chefs de service, chargés de mission) ainsi que des membres du cabinet présidentiel (directeurs de cabinet, directeurs adjoints et conseillers techniques).

Ont également été mobilisés des observations (réunions, visites), des archives (organigrammes des services, arrêtés de nomination, des délibérations de la commission permanente ou de I'assemblée plénière du conseil régional), des documents transmis par les interlocuteurs (PV de réunions) et de la documentation trouvée sur internet (blog d'élus, rapports des conseils économiques et sociaux régionaux ....).

Ce travail a été réalisé dans le cadre d'une recherche coordonnée par Didier Demazière et financée par l'ANR ( ${ }^{\circ}$ ANR-08-GOUV-002) pour la période 2009-2012 sous le titre PRELAT Projet sur les Élus Locaux Au Travail : Gouvernement régional et configuration du travail des élus. Cette recherche porte sur le travail politique dans cinq régions françaises (les auteurs intervenant dans quatre d'entre elles) et sur les mandatures régionales 2004-2010 et 2010-2014. 


\section{La direction générale des services, composante majeure du travail politique régional}

La définition statutaire et la délimitation fonctionnelle des emplois de la DGS (direction générale des services) témoignent l'une et l'autre de la forte imbrication des registres techniques et politiques de la conduite d'une région. Cette configuration trouve notamment sa source dans le flou qui caractérise ces emplois discrétionnaires et dans l'incontournable lien de confiance entre ces directeurs et les présidents de région. Elle se met en place sur fond de technicisation croissante du travail des exécutifs régionaux.

\subsection{Des emplois discrétionnaires aux contours assez flous}

Lappellation initiale de "secrétaire général " reflétait une volonté de séparer nettement les fonctions politiques du travail administratif : le politique décide et l'administration exécute, ce qui valait au responsable des services une forte protection statutaire vis-à-vis du possible arbitraire de l'exécutif (communal à l'origine $\left.{ }^{4}\right)$. Cette configuration a profondément évolué depuis vingt-cinq ans, dans les textes et dans la pratique (Bachelet, 2006).

Avec la mise en œuvre des lois de décentralisation, les textes législatifs et réglementaires ont réaffirmé la primauté des exécutifs territoriaux, traduite dans un nouveau régime statutaire pour les responsables des services des collectivités. La loi du 26 janvier 1984 (article 53) liste des emplois fonctionnels accessibles par la voie du détachement ou par une embauche contractuelle. Pour les départements et les régions, il s'agit du directeur général des services et des directeurs généraux adjoints. Un décret de 1987 précise les conditions d'accès et de carrière 5 .

Ces textes ${ }^{6}$ prévoient des modalités de décharge de fonction qui font qu'un directeur général nommé par voie de détachement peut être amené à quitter son poste pratiquement du jour au lendemain sur la seule volonté du président. De longue date, la jurisprudence du Conseil d'État a établi que le détachement d'un fonctionnaire peut ne pas être renouvelé à son terme par l'organisme d'accueil, voire rompu de manière anticipée, pour tout motif tiré de «l'intérêt du service »; en outre, dans le cas d'espèce des directions générales, elle a sensiblement élargi cette "notion d'intérêt du service en admettant que le

\footnotetext{
4. L'absence de protection aurait pu conduire ces agents à céder à de fortes pressions des élus au risque d'entraîner leurs collectivités aux marges de la légalité.

5. La permanence du recours à des agents contractuels fait que ces derniers peuvent bénéficier, à certaines conditions, d'un contrat à durée indéterminée et se voient reconnaître une sorte de statut spécifique : disposer d'un CDI pour un emploi discrétionnaire a priori d'une durée limitée.

6. Sur les rémunérations de ces agents, voir les grilles indiciaires territoriales (régime indemnitaire spécifique) pour les DGA et DGS de région de plus de 2 millions d'habitants sur le site http://www.emploi-collectivites. fr.
} 
non-renouvellement ou la rupture anticipée puisse être motivé par la "perte de confiance" de l'autorité territoriale en son collaborateur $"{ }^{7}$. Dans les faits, ce motif est systématiquement invoqué par les autorités territoriales. Ainsi, comme le précise l'actuelle responsable du réseau de médiation du SNDGCT (Syndicat national des directeurs généraux des collectivités territoriales), "Avant, on nous appelait pour nous dire : "aidez-nous à conserver notre poste". Aujourd'hui on nous dit: "aidez-nous à en trouver un autre"s ". Aussi, en 2000, ce syndicat a créé un réseau de médiation et de soutien à ses mandants confrontés à des mobilités subies, y compris en vue de l'éventuel contentieux.

La nature de plus en plus politique de ces emplois de direction a clairement renforcé ce caractère discrétionnaire. "On a la confiance du président jusqu'au jour où on ne l'a plus. C'est un métier où l'on est très seul" (entretien avec un DGS de la région R4). Certes les textes posent une délimitation formellement claire des responsabilités : «le DGS (...) [est] chargé, sous l'autorité du président du conseil régional, de diriger l'ensemble des services de la région et d'en coordonner l'organisation " tandis que le directeur général adjoint (DGA) est "chargé de seconder et de suppléer, le cas échéant, le DGS (...) dans ses diverses fonctions $"$ " La réalité est sensiblement moins tranchée, au point que la délimitation de l'administratif et du politique devient très incertaine : "La lère mission du DGS est de faire passer le message et la parole du président sur les dossiers, et de le faire de manière non autoritaire. Il est à l'articulation entre le politique et l'administratif " (entretien avec un DGS de la région R4). En outre, les fiches "métiers " de l'Observatoire de la fonction publique territoriale ${ }^{10}$ indiquent que le DGS "participe à l'explicitation des orientations de la collectivité et à la mise en forme, avec l'équipe politique, d'un projet partagé par toutes les parties prenantes de l'action publique " et qu'il " pilote l'organisation territoriale en cohérence avec les orientations préalablement définies". Cette mission donne lieu à des interprétations différenciées, certaines mettant l'accent sur une conception plutôt administrative, tel ce DGS [R1] confiant qu'il travaille avec « un concept simple : qu'estce qu'une collectivité publique? C'est une entreprise à produire de la décision. Donc il y a de la conception, de la production, de l'évaluation, de la correction. J'ai bâti toute l'administration régionale comme ça»; D’autres donnant un tour nettement plus politique tel «M. qui a voulu sortir la Région de son ronron administratif (...); très réactif, il avait une

7. $C f$. Bazin Jacques, «Emplois fonctionnels », Fonction publique territoriale, Centre national de la fonction publique territoriale, WikiTerritorial : Espaces d'échanges et de partage d'informations autour des collectivités territoriales (http://www.wikiterritorial.cnfpt.fr/xwiki/wiki/econnaissances/view/Notions-Cles/Lesemploisfictionnels), consulté le 11 février 2012.

8. Cf. La Gazette des communes, 7 avril 2008.

9.Voir le décret du 30 décembre 1987 portant dispositions statutaires particulières à certains emplois administratifs de direction des collectivités territoriales et des établissements publics locaus assimilés.

10. Pour le DGS : http://www.observatoire.cnfpt.fr/metiers/?aWQ9NCZhY3Rpb249ZGV0JmlkX21ldGllc j02MzE ; Pour le DGA : http://www.observatoire.cnfpt.fr/metiers/?aWQ9NCZhY3Rpb249ZGV0JmlkX21 ldGllcj02NDE (consulté le 17 octobre 2012). 
vision, il s'emparait d'un dossier clé (...), prenait des positions au risque d'avoir des relations difficiles avec le cabinet" (directrice de l'enseignement supérieur et de la recherche de la région $\mathrm{R} 3$ ).

Pour sa part, sous l'autorité du DGS, le DGA est censé participer «à la définition d'une ligne stratégique de la collectivité ", "diriger les services dans son périmètre et (...) contribuer à la définition des politiques publiques sectorielles». Ainsi que l'avance un DGS lors d'une présentation de ses fonctions à des étudiants, son "rôle a beaucoup évolué depuis 20 ans (...) [pour être] une réelle force de proposition et participer de plus en plus étroitement à la définition stratégique du projet de la collectivité »" ${ }^{11}$ Selon tel autre DGS [R4], "les DGA ont un rôle d'amortisseur ou de tampon entre politique et administratif. Les problèmes politiques ne doivent pas redescendre dans les services. (...) Les DGA doivent être en responsabilité dans une grosse région comme ça ». Ainsi "Le DGA doit maîtriser l'interface entre le politique et le technique car il est sur un poste fonctionnel, il peut être viré du jour au lendemain " (DGS région R4). Mais là encore, la variance des responsabilités effectives s'avère grande : "La fonction de DGA est assez délicate (...) parce que, dans le grand Yalta, certains se retrouvent avec trop de choses quills ne peuvent pas toutes gérer et d'autres, c'est un peu vide, soit trop, soit trop peu " (directeur d'un service, région R3).

Cette évolution générale vers une implication plus forte de la DGS dans la définition des projets de la collectivité territoriale conforte l'hypothèse (Demazière, Le Lidec, 2008, p. 141) selon laquelle "les frontières entre positions politiques et administratives de direction sont de plus en plus poreuses, favorisant la constitution d'un monde commun caractérisé notamment par le partage des responsabilités dans la décision et son corollaire, le risque électoral».

À notre sens, cette porosité est d'autant plus probable que demeure une incertitude sur la définition du rôle in situ de tel DGS ou de tel DGA. Ainsi, à leur manière, ces emplois de direction relèvent eux aussi des " métiers flous $\|^{12}$ qui prolifèrent au sein de la fonction publique territoriale au fil de l'imbrication des compétences institutionnelles et des financements publics (Jeannot, 2005). La place que tient la confiance dans le lien qui unit un président de région et son (sa) DGS atteste de l'incertitude qui entoure la définition et les conditions d'exercice de ces fonctions de direction générale.

11. "Le DGS : clé de voûte des collectivités », Conférence du 3 mars 2010 réalisée dans la cadre du Master 2 de Management Public Territorial de l'IEA de Lille, cf. http://www.regards-croises-de-mt.sitew.com/files/ users/3/0/8/3/9/4/6/CR_Conference_IAE_du_3mars2010_Le_DGS.pdf.

12. Ces «métiers» se caractérisent par des missions dont la définition est assez vague, des positions d'empois parfois bricolées et, pour les personnes, des parcours influencés par la sphère politique; 


\subsection{L'importance du lien de confiance entre le président de l'exécutif et le (la) DGS}

En charge du fonctionnement de l'institution régionale, toute direction générale voit sa solidité et sa durée être suspendues à l'instauration d'un lien de confiance entre le président et le(s) responsables des services. Cette relation est d'autant plus cruciale que la fonction de DGS est devenue plus stratégique, appelée qu'elle est à contribuer explicitement à l'élaboration du projet régional et à son accommodement constant aux circonstances, tout au long du mandat de l'assemblée. Cette confiance entre le président et son DGS doit être régulièrement réassurée et confortée au fil des épreuves auxquelles est confronté tout exécutif. Pour autant, elle est difficile à définir positivement puisque ce sont d'une part les conditions d'entrée en fonction et d'autre part, les conséquences du délitement de ce lien qui permettent d'en apprécier l'importance :

- Les DGS sont recrutés au vu de relations construites de longue date entre les protagonistes $^{13}$;

- La perte de confiance se traduit en effet par le départ quasi-systématique du directeur concerné, rappelant que, quelle que soit l'imbrication du politique et du technique, in fine il n'y a qu' "un seul patron [le président]" (un DGS de la région R3).

Plus généralement, la contribution de la DGS au travail politique de l'exécutif ne peut se définir par les seuls critères du statut des emplois de cadre dirigeant, de l'expertise professionnelle de ses membres ou encore du management organisationnel qu'elle doit assurer. L'assemblage de ces dimensions est mis au service d'un pilotage stratégique qui articule de constantes exigences de court terme - portées notamment par les attentes des élus et notamment du président et de son cabinet - à la mise en œuvre du projet de mandature régulièrement ré-ajusté. Le DGS est le premier garant de cette délicate cohérence.

À ce titre, avec la complexification croissante des missions des régions, sa légitimité se joue sur plusieurs registres clés :

- Etre un facilitateur des multiples projets qui structurent l'action publique régionale en s'appuyant sur les expertises plus techniques et sectorielles de ses DGA, véritables forces de propositions pour la définition des politiques publiques de la région : celle-ci élabore des orientations thématiques pluri-annuelles - plan régional de développement de la formation, schéma prévisionnel des formations sanitaires et sociales, schéma régional de développement économique, schéma régional d'aménagement du

13. Comme en témoignent les exemples suivants : les deux DGS successifs de telle région [R4] avaient été membres, plusieurs années auparavant, des cabinets ministériels de l'actuel président de région ; tel autre, dans une région voisine, avait non seulement été un collaborateur de poids du cabinet présidentiel mais, en outre, était issu du réseau de relations locales du président en question. 
territoire ... - etc. ${ }^{14}$, sachant que « dans la période des schémas (...), ce sont les services qui avaient l'initiative " (DGA de la région $\mathrm{R} 3$, antérieurement membre du cabinet présidentiel).

- Sécuriser les procédures dans un environnement de plus en plus incertain, ce qui signifie non seulement "faire tourner la boutique ", attente constante des élus, en tenant un rôle de contrôle juridique et financier, mais aussi assurer la coordination externe avec des partenaires d'autant plus diversifiés que la clause de compétence générale dont jouissent toutes les collectivités territoriales leur permet d'intervenir dans de nombreux domaines en mobilisant des partenariats ad hoc. Il reste que l'État, au premier chef la préfecture de région et son secrétariat régional aux affaires régionales (SGAR), demeure un interlocuteur incontournable : environ la moitié du budget régional est dépensé avec l'État, à travers notamment les contrats de projet.

- En outre, avec ses collaborateurs directs, il doit être en mesure d'assumer une fonction de veille juridique, financière et managériale (révéler de "bonnes pratiques ») afin de prévenir des risques techniques susceptibles de se transformer en aléas politiques majeurs, faute par exemple d'une réorientation précoce de tel ou tel projet de la région.

L'activité de la direction générale des services s'inscrit donc dans un mode de régulation et de décision nécessairement collaboratif ; il se démarque d'un modèle hiérarchique faisant du DGS le point de passage obligé de la circulation ascendante et descendante de l'information, même si certains DGS s'efforcent de perpétuer une certaine forme de centralité : "Toutes les décisions me remontent : y compris sur les détails, il n'y a pas grand chose qui méchappe " (un DGS de la région R1). Il vise ainsi à limiter les incessants processus de débordement de sa capacité de suivi et d'anticipation. Il reste que le travail politique régional est une figure collective et multiforme au contenu fortement relationnel : à ce propos, le couple DGS-président de région illustre bien que ce travail politique est " ̀̀ la fois rationalisé et personnalisé " (Demaziere, Le Lidec, 2008, p. 141). Ainsi "le président est un homme qui recherche l'efficacité, il prend une décision que quand il a l'exhaustivité du dossier : le pour et le contre du dossier. Après son DGS, l'administration, il l'organise comme il l'entend" (un DGS de la Région R1).

\subsection{Technicisation et professionnalisation du travail politique régional}

Comme l'illustre le tableau 1, la manifestation la plus évidente de cette tendance tient à la création de postes de DGA au tournant de l'an 2000. Ainsi dans l'un des conseils régionaux étudiés, la création des premiers postes de DGA n’est intervenue qu'en 1999. Jusque-là, le DGS gérait seul en direct le pilotage et l'animation d'une douzaine de directions. Dans une autre région, leur nombre est passé de deux initialement (en 1998),

14. Cette «mise en plan" (Buisson-Fenet, Verdier, 2011) est adossée à une intense activité de consultations, concertations, contractualisations, et programmations, pilotée par le DGS et les DGA. 
avec une répartition très simple entre d'un côté le social et de l'autre l'économique, à six aujourd'hui, chaque DGA chapeautant quelques directions rassemblées selon des principes assez hétérogènes (voir ci-dessous) ${ }^{15}$. Plus récemment enfin, dans une 3ème région, le nombre de DGA a cru de huit à treize, répartis entre des fonctions administratives (logistique, ressources humaines, etc.), des missions opérationnelles (direction de l'économie et de l'innovation, etc.) et des missions d'interface (vie institutionnelle et communication, médiation).

La division du travail de conception s'est ainsi accrue pour renforcer le poids de ces hauts techniciens de l'action régionale. Un DGS [R1] raconte qu'il «voulait une organisation très déconcentrée avec une forte autonomie aux DGA, je préfere manager les équipes comme ça, c'est mon tempérament (...) Je l'ai fait avec mes DGA et j'ai poussé mes DGA à le faire avec leurs directeurs ». D'ailleurs l'apparition et l'extension de cette nouvelle strate au sein de la hiérarchie administrative a été vécue par certains " grands élus " (que ce soit des présidents de commission - PDC - ou des vice-présidents - VP) comme le vecteur d'un affaiblissement de leur pouvoir politique de conception et de conduite de l'action régionale : l'un d'entre eux [R3] est allé jusqu'à qualifier les DGA de «couche administrative nuisible » sachant que dans la configuration antérieure, VP et/ou PDC avaient souvent pris l'habitude d'entretenir des relations directes avec les services. Aujourd'hui, de telles pratiques les mettent souvent en tension avec ces nouveaux hauts fonctionnaires régionaux, soucieux de voir les élus respecter la hiérarchie administrative, quitte à rappeler qu'eux seuls disposent, dans leurs domaines d'intervention, de la "signature ", c'est-à-dire d'un pouvoir d'engagement réel délégué par le président.

\section{Tableau 1}

\section{Evolution du nombre de DGA et de pôles dans les quatre régions étudiées}

\begin{tabular}{|l|l|c|c|c|c|c|c|c|c|}
\hline \multicolumn{2}{|l}{ Régions/Années } & $\mathbf{1 9 9 8}$ & $\mathbf{2 0 0 0}$ & $\mathbf{2 0 0 4}$ & $\mathbf{2 0 0 6}$ & $\mathbf{2 0 0 8}$ & $\mathbf{2 0 1 0}$ & $\mathbf{2 0 1 1}$ & $\mathbf{2 0 1 2}$ \\
\hline \multirow{2}{*}{ R1 } & Fonctions administratives & 0 & 0 & 0 & 2 & 2 & 2 & 2 & 2 \\
\cline { 2 - 11 } & Missions opérationnelles & 2 & 2 & 2 & 2 & 2 & 2 & 3 & 2 \\
\hline \multirow{2}{*}{ R2 } & Fonctions administratives & 2 & 2 & 2 & 2 & 2 & 2 & 4 & 6 \\
\cline { 2 - 11 } & Missions opérationnelles & 3 & 3 & 3 & 4 & 4 & 5 & 5 & 5 \\
\cline { 2 - 11 } & Missions d'interface & & 1 & 1 & 1 & 1 & 1 & 3 & 2 \\
\hline \multirow{2}{*}{ R3 } & Fonctions administratives & 0 & 1 & 1 & 2 & 0 & 1 & 1 & 1 \\
\cline { 2 - 11 } & Missions opérationnelles & 2 & 2 & 2 & 2 & $3 / 4$ & 5 & 5 & 5 \\
\cline { 2 - 10 } & Missions d'interface & & & & & 1 & & & 1 \\
\hline \multirow{2}{*}{ R4 } & Fonctions administratives & 0 & 1 & 1 & 1 & 1 & 1 & 1 & 5 \\
\cline { 2 - 10 } & Missions opérationnelles & 0 & 3 & 5 & 5 & 5 & 5 & 5 & 5 \\
\hline
\end{tabular}

Sources : organigrammes des conseils régionaux.

15. Les transferts de compétence liés, en 2004, à " l'acte II » de la décentralisation ont accéléré ce processus avec d'une part, l'élaboration du schéma prévisionnel des formations sanitaires et sociales ainsi que du schéma régional de développement économique "; d'autre part, les transferts aux régions des techniciens, ouvriers et personnels de service des lycées, occasionnant un quadruplement de leurs effectifs. 
Pour les présidents et leurs cabinets, le mode de délimitation des divers pôles thématiques qui composent la DGS constitue un moyen assez puissant pour peser sur l'activité tant des VP que des directeurs généraux. Dans nombre de conseils régionaux, le champ des délégations accordées aux VP ne correspond pas à celui des pôles placés sous la responsabilité des DGA. Il n'est donc pas rare qu'un VP soit amené à se coordonner avec au moins deux DGA pour exercer sa faculté d'initiative. Cette dissociation des géographies de l'administration et de l'exécutif rend plus difficile de la part d'un VP l'exercice d'un pouvoir d'influence et a fortiori d'un pouvoir de direction et de contrôle des services. En outre, les VP ne disposent pas de la capacité à recruter les hauts responsables de la région. À tout le mieux, ils sont consultés par la DGS et le cabinet, parties prenantes majeures de ces décisions stratégiques. De plus, cette dissociation revêt un autre avantage majeur pour le président. Il est ainsi en mesure de modifier l'organigramme de la région et de restructurer les services sans être obligé d'interagir étroitement avec les VP puisque leurs délégations ne sont pas directement affectées.

Pour autant, cette affirmation de l'autorité présidentielle n'est pas en soi un gage de stabilité des organisations. En attestent, entre autres, de fréquents changements de titulaires de ces hauts postes fonctionnels et, à un moindre degré, de la configuration en pôles de la DGS. Ces révisions périodiques, évidemment plus profondes en début de mandature et en particulier en cas d'alternance politique, s'avèrent parfois très conflictuelles. Elles témoignent de la difficulté à construire des " ordres locaux " (Friedberg, 1993) qui confortent l'assise de la jeune institution régionale. Cette recherche d'ajustements efficaces et légitimes entre services et exécutif élu sollicite assez intensivement la capacité de coordination interne de l'action régionale, d'autant que chaque partie prenante est fondée à revendiquer sa part de légitimité professionnelle. De plus en plus souvent, les VP font la preuve d'un exercice professionnalisé de leurs mandats, situation souvent reconnue par les DGA tel celui-ci [R3] : "Les élus sont assez bons et compétents dans leur domaine. Je n'ai pas besoin d'aller dans toutes les commissions car les élus sont de plus en plus au point». La concurrence pour exercer un leadership n'en est que plus exigeante.

Par-delà des références légales, des environnements institutionnels communs et des choix d'organigramme voisins, deux constructions distinctes du travail politique régional émergent et contribuent à formater les positions et les modes d'exercice des DGS et des DGA. 


\section{La gestion des postes de DGS et DGA, entre carrières administratives et régulation politique}

Dans un fort contexte d'incertitude, le duo formé par le cabinet du président et la DGS s'efforce de produire la capacité d'intégration nécessaire à la fabrique au long cours et au quotidien des politiques des diverses régions étudiées, par delà les cloisonnements sectoriels, les clivages politiques internes à la majorité régionale ou encore les intérêts des différents territoires. Au-delà des héritages politiques propres à telle ou telle collectivité régionale (Mattina, 2004), comprendre les fondements des spécificités organisationnelles des régions, ici de leur direction générale, nécessite de les situer dans un cadre plus large, à savoir les relations entre les cinq pôles constitutifs de la régulation régionale : le président et son cabinet ; la DGS ; les services ; les vice-présidents ; les groupes partisans et les commissions de travail. Une première configuration repose sur la prééminence surplombante de la fonction présidentielle (le chef de l'exécutif et son cabinet), alors que la DGS peine à faire reconnaître sa légitimité comme composante pleine et entière du travail politique régional ; la seconde promeut une DGS que l'on peut qualifier de "gouvernementale ", dans le cadre d'un présidentialisme " tempéré ».

\subsection{Pilotage politique de l'institution régionale : délicate affirmation de l'autonomie de l'administration}

Dans cette configuration, la DGS joue le rôle de variable d'ajustement vis-à-vis des tensions internes récurrentes dont témoigne l'instabilité - au moins relative - de ses membres et de sa configuration. En outre, l'accès à ces places de haut fonctionnaire régional s'inscrit dans un marché interne où se conjuguent proximité relationnelle et distinction hiérarchique entre le politique et la sphère administrative, attestant de la volonté présidentielle de se garantir, en toute circonstance, l'exercice plein et entier de son pouvoir discrétionnaire.

\subsubsection{Une DGS sous tutelle}

Ce positionnement se manifeste en premier lieu par la primauté du cabinet sur la direction générale : celui-ci est explicitement chargé de la mise en œuvre du projet présidentiel et d'y ajuster la marche de l'administration. C'est ainsi qu' " avant pendant longtemps, le directeur de cabinet avait la prééminence sur le DGS» (un DGS de la région R3) ; en outre, "le DirCab joue un rôle fort, même si on a eu ici des DirCab très différents les uns des autres" (directeur de cabinet adjoint de la région R3).

Chargé de promouvoir autant que faire se peut la personne présidentielle, le cabinet est composé de jeunes "techniciens ", relativement stables et proches du président ; leurs missions respectives sont structurées en fonction du périmètre tant des délégations que des administrations : de facto en charge de la cohérence de l'action régionale, le cabinet 
veille au respect des orientations du projet présidentiel (" projet de mandature »); chaque conseiller technique est censé être le garant du respect de la ligne présidentielle. Il est ainsi amené à entretenir une relation directe avec les services opérationnels, dont sourd le risque récurrent de tensions vives entre l'entourage du président et la DGS. Si en théorie, ce schéma organisationnel a été admis par les DGS, sa mise en œuvre n’a pas été toujours aisée tant il a été traversé de crises, parfois ouvertes.

Ainsi dans l'une des régions, les DGS se sont succédé à un rythme assez soutenu (cinq titulaires en treize ans), témoignant ainsi de leur rôle de fusible institutionnel alors que les directeurs de cabinet étaient relativement stables - trois - dont l'un a démissionné de son propre chef pour rejoindre un autre exécutif territorial. À ce propos, soulignons que les courts-circuits ont aussi été l'œuvre directe de tel ou tel DGS n'admettant plus les intrusions répétées de la présidence et de certains VP dans le fonctionnement des services, jugeant qu'elles rendaient encore plus difficile la mise sur pied de procédures opposables qui, à ses yeux, justifiait les exigences croissantes de professionnalisme formulées à l'encontre de l'ensemble des agents : «ça été le soubait de X [ex DGS de R3] de rationaliser (...) et de sécuriser autant que faire se peut l'administration et d'organiser des rapports de force plus équilibrés entre le point de vue exprimé par les politiques et ce quil est possible de faire dans une administration ". La brutalité de la séparation a été à la hauteur des liens quasi-affectifs qui unissaient les deux protagonistes, l'un tentant « le tout pour le tout en donnant sa démission au président, pensant que le président ne l'accepterait pas. Mais il n'a pas accepté qu'on lui fasse du chantage, considérant que ceétait du chantage " (DGA de la région R3 sur trois mandatures).

Ce mode de régulation, au final très centralisé, très politique et très personnalisé a évidemment son prix, à savoir la concurrence réitérée de diverses légitimités (outre le cabinet, les VP et leurs collaborateurs, les DGA et/ou le DGS) assortie de la conclusion d'alliances assez instables et d'une portée incertaine : par exemple celle nouée entre le chargé de mission de tel VP et un directeur de service pour contourner le DGA compétent et être ainsi en mesure d'influer sur le cabinet; ou encore des liens privilégiés entre un DGA et le cabinet pour échapper au contrôle du DGS, etc.. Cette menace récurrente d'une instabilité organisationnelle a conduit, au début de la précédente mandature, à instaurer une instance de pilotage propre à chaque délégation et réunissant très régulièrement les diverses parties prenantes internes des politiques concernées : le VP et ses collaborateurs, les DGA (et les directeurs de services en tant que de besoin), les conseillers délégués et toute personne jugée nécessaire compte tenu d'un ordre du jour préalablement établi. Cet ordonnancement n'a pas résisté au lent travail de sape des concurrences et des alliances (parfois improbables), ni aux chocs engendrés par des démissions brutales de DGS (voir ci-dessus) assorties de changements d'organigramme relançant les luttes sur le périmètre des différents pôles constitutifs de la DGS : "Cet organigramme est déséquilibré; $X(D G A)$ a happé les sources de distribution d'argent de la 
Région et absorbé tout ce qui bongeait. Y [autre DGA antérieurement supérieur du précédent] a récupéré des bouts de bois" (Directeur de service de la région $\mathrm{R} 3$ ).

Tableau 2

DGS (directeurs généraux des services) rencontrés

\begin{tabular}{|l|c|c|}
\hline Régions & Profil / Formation initiale & Postes précédents \\
\hline R1 & IRA droit public & Préfecture, puis chef de service Conseil Général \\
\hline R1 & Enarque préfectorale & Directeur de cabinet, puis Préfecture \\
\hline R3 & Enarque IGA & Ministère de l'Intérieur puis DGA Région \\
\hline R3 & Docteur économie des transports Ministère des Transports & Directeur de service et DGA Région \\
\hline R3 & Enarque préfectorale & Préfecture puis DGS Région \\
\hline R4 & Enarque Conseil d'État & Commission européenne, cabinets ministériels \\
\hline R4 & Enarque préfectorale & Préfecture, cabinets ministériels, Haut-commissariat de la \\
& & République \\
\hline
\end{tabular}

Source : auteurs.

Sigles : IRA (Institut régional d'administration), IGA (Inspection générale de l'administration), DGS (directeur général des services), DGA (directeur général adjoint).

\subsubsection{Un marché interne pour accéder aux postes de DGA}

Dans les régions relevant de ce mode de gouvernance ${ }^{16}$, la régulation de l'accès aux postes de direction relève d'une logique de marché interne. Elle associe des critères d'expérience professionnelle au degré de proximité avec des élus influents et surtout le cabinet ${ }^{17}$. De ce fait, les anciennetés de hauts responsables peuvent être très élevées, au-delà même du quart de siècle. En comparaison, les profils de DGA sont hétérogènes (recrutement dans des directions de la région, passage par le cabinet ${ }^{18}$, d'autres collectivités locales, ...) avec une moindre présence de membres de la haute fonction d'État ( $c f$. tableau 3). Si quelques DGA ou directeurs sont recrutés à l'extérieur de l'institution, ces mobilités se réalisent dans un cercle très fermé au sein de réseaux politiques ou d'institutions très proches de la région. Ces parcours largement internes à l'institution régionale rendent encore plus floues les frontières entre élus, cabinet et services.

16. Ce modèle correspond aux cas des régions $\mathrm{R} 2$ et $\mathrm{R} 3$.

17. Ce qui n'interdit pas de recourir au recrutement externe si n'émergent pas des candidatures internes probantes.

18. Si dans l'une des régions en cause [R3], ces mobilités internes ne s'opèrent que dans un sens - deux conseillers techniques deviennent DGA, un directeur de cabinet devient DGS -, dans l'autre [R2], les mobilités s'effectuent dans les deux sens : l'un des DGA devient directeur-adjoint du cabinet avant de redevenir DGA tandis qu' un autre DGA passe directeur de cabinet, tout comme un directeur de cabinet devient DGA. Les allers-retours sont donc nombreux entre les services et le cabinet. 
À chaque modification de l'organigramme, les responsables qui restent au sein de l'institution régionale doivent être " recasés ". On assiste à une "sorte de jeu de chaise musicale ". La logique qui prime est celle de l'addition qui conduit parfois à l'augmentation du nombre de DGA au fur et à mesure des réaménagements ${ }^{19}$ : " c'est plus facile de redistribuer les pôles que de les resserrer" (entretien avec un DGA de la région R2). Il faut replacer tout le monde avant de trouver de la cohérence dans l'organisation des pôles ou avant de recruter à l'extérieur. En cas de conflit ouvert (entre un DGA et un VP, entre un DGA et ses directions), les problèmes sont plus difficilement résolus puisqu'il faut trouver une solution interne (changement de pôle). Quand les tensions deviennent trop grandes, il faut trouver une porte de sortie externe, via notamment des institutions satellites de la région qui offrent des postes de substitution. Ainsi avec " des logiques de recrutement qui sont plus internes, on n'est pas dans un exercice consistant à faire coller un profil avec la capacité de diriger un secteur mais dans une logique de répartition des places. Il faut caser les gens" (actuel DGS de la région R3).

Dans ce contexte fait d'incertitudes diverses, ce marché interne fonctionne selon une logique qui s'apparente à celle décrite par la théorie économique en termes de "tournois $»^{20}$ (Lanfranchi, 1992) mais sous l'empire d'une rationalité pour le moins limitée : dans le cadre d'une concurrence inter-individuelle sévère pour l'accès aux postes de la DGS et en vue d'assurer un périmètre suffisant au pôle thématique visé, chaque prétendant s'efforce de construire un réseau de relations et d'influences suffisamment puissant pour être en mesure de saisir les opportunités qui s'offrent ; dès lors, les positions acquises sont loin d'être assurées. Ainsi dans l'une des régions emblématiques de cette configuration, l'un des deux DGA du début de la décennie - couvrant donc la moitié du champ des politiques régionales - co-existe aujourd'hui avec deux ex-subordonnés alors chefs de service qui disposent aujourd'hui l'un et l'autre d'un périmètre sensiblement plus important que celui de leur ex-DGA qui ne fut pas loin d'être totalement placardisé il y a trois ans. Plus généralement, dans le cas des DGA qui étaient auparavant directeurs, se font jour des problèmes de positionnement vis-à-vis des directeurs qui les ont remplacés, quand ils ne cumulent pas parfois la fonction de responsable de service avec celle de DGA. Leur légitimité interne les conforte dans une gestion plus directe des dossiers. Ils ne se rangent pas spontanément dans le rôle d'animateur de projets diversifiés qui leur revient en principe.

19. Certains gardant leur grade/titre de DGA même s'ils ne sont pas forcément à la tête d'un pôle ou d'une délégation générale adjointe.

20. En prenant en compte les différences relatives et non absolues de performance individuelle comme le fait la théorie économique standard, l'approche des marchés internes en termes de tournois cherche à expliquer que de faibles écarts de capacité (ou même la chance) entraînent une considérable différence de gain : comme le champion olympique qui l'emporte pour un rien, les personnes finalement promues aux très rares postes de directions le sont souvent pour de très faibles compétences distinctives vis-à-vis des autres candidats. 
Dans une sorte de paradoxe, ces concurrents comme l'ensemble des responsables de services susceptibles de devenir DGA partagent néanmoins un référent commun : faisant carrière dans ce conseil régional, pouvant avoir servi des exécutifs de couleurs politiques parfois différentes, ils cherchent à stabiliser le fonctionnement de l'institution régionale. Dans leurs propos, revient fréquemment l'expression de "sécurisation des procédures régionales " ou encore de "professionnalisation du travail de l'administration", notamment dans la composante réalisée à la demande directe de l'exécutif. Le souci des services de mettre à distance les élus membres de l'exécutif exprime avant tout une demande de respect et de reconnaissance de la valeur et de la légitimité intrinsèque de leur travail afin de limiter le poids de l'arbitraire politique. Dans l'une des régions étudiées, la volonté de rompre avec des pratiques clientélistes favorisées dans le passé par des majorités relatives se traduit par l'imposition progressive, de la part des DGS successifs, de critères d'instruction des dossiers "signalés " par tel ou tel élu influent. En témoigne également la création d'une inspection générale des services.

\subsection{Une DGS « gouvernementale » : une légitimité avant tout externe}

Tranchant nettement avec le modèle précédent, cette configuration vise à répondre à l'incertitude qui pèse sur tout exécutif régional "en associant des cercles divers à la préparation des décisions" (directeur-adjoint de cabinet de la région R4) : ce mode de gouvernance a produit des apprentissages institutionnels qui ont progressivement assis la légitimité de la DGS tout en confortant la capacité d'arbitrage présidentielle : " ni omniprésent ni omnipotent, tout en ayant un certain ego, mais pas celui de se soucier qu'aucune tête dépasse derrière lui " (membre du cabinet de la région $\mathrm{R} 4$ ), "le président travaille en râteau : il a besoin de l'avis de son directeur général, de son vice-président, de son cabinet pour décider" (autre membre du cabinet de la région R4). En outre, l'assise de la DGS est renforcée par le recrutement essentiellement externe de membres largement issus des grands corps de l'État.

Mais ces conseillers ont avant tout la charge de construire une capacité de réaction politique à court terme, sachant que les DGA « ont leurs limites, tenant aux portefeuilles immenses quills gèrent et, surchargés, ne sont pas en mesure d'intervenir sur des événements externes imprévus " (DirCab de la région $\mathrm{R} 4$ ).

\subsubsection{Le DGS, garant des cohérences politiques et organisationnelles internes à la région}

Ce positionnement a été progressivement acquis en traversant diverses épreuves. Les plus anciennes remontent aux temps de majorités relatives lors desquelles s'est expérimenté un présidentialisme tempéré par l'effectivité d'une forme assez originale de parlementarisme régional. Si depuis lors, la DGS s'est imposée comme l'instance légitime de la conduite des politiques de la région, elle l'a fait en maintenant un processus actif de délibération par l'assemblée et, à un moindre degré, de ses commissions de travail. Le passage de certains élus du rôle de président de commission à celui de vice-prési- 
dent dans le même domaine de compétence a, semble-t-il, favorisé une telle transition. Cette configuration d'ensemble atteste d'un fonctionnement plus " parlementaire " que dans l'autre configuration régionale.

Cependant, par-delà le soin porté aux procédures de délibération, l'instauration d'une telle régulation ne résulte pas d'un mode d'exercice prêt à l'emploi. Ainsi dans la région sans doute la plus emblématique à cet égard, une épreuve fondatrice majeure est intervenue à l'occasion de l'installation d'une nouvelle majorité. Désireux d'affirmer sa primauté au titre d'un changement politique se voulant profond, l'exécutif et notamment les nouveaux VP ont développé des pratiques résolument intrusives dans le fonctionnement interne des services ${ }^{21}$. Fortement contradictoire avec les pratiques qui avaient jusqu'alors prévalu, ce brutal changement de cap a débouché sur une très vive tension qui a déclenché un mouvement de grève assorti d'un rassemblement du personnel dans le grand hall du conseil régional. À l'époque, certains élus ont en effet été jugés excessivement irrespectueux à l'égard de techniciens qu'ils avaient tendance à traiter comme "des courtisans », pour reprendre les termes d'une directrice de service de l'époque, plutôt que comme des partenaires reconnus du travail politique à élaborer conjointement et, pour ce qui les concerne, au titre de leur légitimité technique. Afin de sortir de ce conflit ouvert, un petit groupe de travail paritaire rassemblant des élus et des représentants des personnels administratifs a élaboré, sous l'égide d'un nouveau DGS à la très forte réputation tant professionnelle que politique, une charte des relations entre élus et services; cette dernière a fait de la DGS l'instance de règlement des éventuels conflits entre des membres des deux parties

\subsubsection{Le recrutement des DGA sur le marché externe}

Depuis une dizaine d'année, ces conseils régionaux (ce modèle correspond aux cas des régions $\mathrm{R} 1$ et $\mathrm{R} 4$ ) ont pris l'habitude de recruter ${ }^{22}$ assez systématiquement de hauts fonctionnaires, d'État en particulier, dont le parcours témoigne non seulement d'une communauté d'idées et de valeurs avec les élus de l'exécutif ${ }^{3}$ mais aussi de leur aptitude à accommoder la régulation managériale et des jeux politiques toujours complexes : « $\mathrm{I} l y$ a un recrutement externe en haut (...) les DGA ont un haut niveau d'expertise" (DGS de la Région R4). Toutefois, les canaux de recrutement du DGS et des DGA differrent.

21. Par exemple, tel membre de l'exécutif intervenant sur l'organisation d'un service sans tenir compte des responsabilités des divers échelons hiérarchiques (DGS, DGA, directeur, chef de service ...) ou se préoccupant a priori dans ses moindres détails de la mise en oeuvre d'une résolution politique.

22. Ces recrutements se sont le plus souvent par le biais de réseaux professionnels ou informels mais de plus en plus par l'entremise de cabinets de recrutement spécialisés " chasseurs de tête ".

23. Ainsi quelques années auparavant, tel DGS avait été membre du cabinet d'un ministre devenu depuis lors président de région. 
Si de nombreux DGS sont énarques (anciens préfet ou sous-préfets, conseiller d'État ou inspecteur général de l'administration) à l'instar de certains DGA (voir tableau 2), leur arrivée résulte toujours de l'activation d'un lien fort existant au préalable, notamment une commune expérience en cabinet ministériel ; c'est ainsi que tel DGS a conseillé le président lorsqu'il exerçait des fonctions gouvernementales et devient aussi son directeur de cabinet dans la dernière phase d'une mandature. Pour autant, ce DGS n'a jamais obtenu le pouvoir de composer à sa discrétion son équipe de DGA, le président et son cabinet cherchant ainsi à garantir leur pouvoir d'arbitrage.

Tableau 3

DGA rencontrés

\begin{tabular}{|c|c|c|c|}
\hline Régions & Profil/ Formation initiale & Postes précédents & Pôles \\
\hline R1 & Enarque & Sous-directeur Mairie de Paris & Emploi Formation Culture Sport \\
\hline R1 & Chercheur & CNRS ARPE & Aménagement \\
\hline R2 & Professeur proviseur & Lycée, Rectorat, Directeur région & Education \\
\hline R2 & Sciences Po INET Administrateur & Directeur RH Communauté urbaine puis Région & Economie Formation Recherche \\
\hline R3 & Master Urbanisme & Consultant Réseaux européens & Relations Internationales \\
\hline R3 & Master Economie & Cabinet Région & Emploi Formation \\
\hline R3 & $\begin{array}{l}\text { Faculté de sciences économiques } \\
\text { Master Développement territorial }\end{array}$ & Conseil Général & Politiques territoriales \\
\hline R3 & Master Gestion publique & Cabinet Région & Education puis ressources \\
\hline R3 & $\begin{array}{l}\text { Docteur Economie des transports } \\
\text { Ministère des Transports }\end{array}$ & Directeur Région & Aménagement Transports \\
\hline R3 & Enarque IGA & Ministère de I'Intérieur & Emploi Formation \\
\hline R4 & Enarque IGAENR & Ministère de l'Education & Education \\
\hline R4 & Inspecteur du travail & $\begin{array}{l}\text { Directeur régional du travail, de l'emploi et de la } \\
\text { formation professionnelle }\end{array}$ & Emploi Formation \\
\hline R4 & TPE-Ponts & $\begin{array}{l}\text { Ministère de l'Equipement Directeur grande } \\
\text { école }\end{array}$ & Transports puis Territoires \\
\hline R4 & TPE-Ponts Urbaniste en chef de l'État & $\begin{array}{c}\text { Ministère de l'Equipement Communauté } \\
\text { urbaine }\end{array}$ & Transports \\
\hline R4 & Personnel administratif Université & Directeur Services Région & Economie \\
\hline R4 & Agrégation, Maîtrise & $\begin{array}{l}\text { Professeur, Directeur Etablissements culturels, } \\
\text { Directeur Régional des Affaires Culturelles }\end{array}$ & Culture \\
\hline
\end{tabular}

Source : Auteurs.

Sigles : ARPE (Agence régionale pour l'environnement), INET (Institut national des études territoriales), IGA (Inspection générale de l'administration), IGAENR (Inspection générale de l'administration de l'Éducation nationale et de la recherche), TPE (Travaux publics de l'État). 
Le recrutement des DGA s'appuie très fréquemment sur l'intermédiation d'un cabinet de " chasseurs de tête ", notamment sur l'un d'entre eux, plus particulièrement dédié aux cadres supérieurs de la fonction publique territoriale entre 2002 et 2012. De la « short list ", le DGS et le directeur de cabinet établissent un classement de deux ou trois personnes, ou encore proposent au président un candidat qui doit alors obtenir la confirmation du chef de l'exécutif ( $c f$. tableau 3).

Cette mobilisation d'un haut niveau d'expertise est notamment motivée par la volonté de développer une stratégie efficace de négociation avec l'État, dans un contexte relationnel souvent assez conflictuel. Dans deux des régions étudiées, tous les DGA sont recrutés à l'extérieur de l'institution voire parfois même de l'espace géographique régional (cf. tableau 3). Ils occupaient tous des postes stratégiques en rapport avec les missions qu'ils occupent au sein de la DGS chez des partenaires importants de la région, que ce soit au niveau de l'État (Rectorat, Inspection générale de l'administration de l'Éducation nationale et de la Recherche, direction régionale des Entreprises, de la Concurrence, de la Consommation, du Travail et de l'Emploi, direction régionale du Travail, de l'Emploi et de la Formation professionnelle, direction régionale des Affaires culturelles, ministères des Transports ou de l'Agriculture) ou auprès d'autres institutions (Communauté urbaine, chambre régionale de Commerce et d'Industrie, chambre régionale des Comptes, Agence régionale pour l'Environnement).

Ainsi dans l'une de ces régions [R1], sur les cinq DGA en poste en 2011, trois sont des énarques, dont deux sont magistrats de la Chambre régionale des Comptes. Ces deux derniers sont responsables de deux pôles administratifs ; cela permet, selon le DGS, de sécuriser la région face aux différentes procédures de contrôle et d'audit qu'elle pourrait subir. Les deux autres DGA sont respectivement ingénieur des Mines et chercheur au CNRS en détachement. Jusqu'à 2006, il n'y avait que deux DGA dans cette région. Dans le champ de l'économie ou des affaires européennes, comme dans certains domaines techniques tels les transports ou l'agriculture, le recours à de hauts profils (des X-Ponts, ingénieurs des Mines, centraliens ou ingénieurs ENGREF - École nationale du génie rural, des eaux et des forêts - en particulier) concerne également des niveaux de responsabilités inférieurs (chefs de services). Afin de mieux maîtriser les affaires européennes, plusieurs régions ont ainsi cherché à recruter des directeurs ayant une solide expérience du montage des dossiers de fonds européens, qu'ils proviennent du ministère de l'Agriculture, de réseaux européens, du Secrétariat général des Affaires européennes de Matignon ou qu'ils aient travaillé à la Commission européenne comme expert national détaché. Dans les directions des Lycées, les responsables des services viennent majoritairement du ministère de l'Éducation ou de celui de l'Agriculture (lycées agricoles). Enfin dans d'autres directions, il a été fait appel à des hauts fonctionnaires chevronnés de la métropole régionale. Si ces recrutements attestent de l'attractivité de la région (" des gens qui étaient numéro un dans le secteur où 
ils étaient ont accepté de venir à la région" (DGS de la Région R4), ils revêtent aussi une signification plus gestionnaire.

Non seulement ils confortent la légitimité de la DGS vis-à-vis des services ${ }^{24}$ mais, en instaurant comme règle d'embaucher à l'extérieur les hauts responsables de l'administration, ils rendent vain tout esprit de compétition au sein des services : les mobilités ascensionnelles vers les postes de direction générale ont vocation à se réaliser hors de la collectivité régionale ${ }^{25}$.

Dans cette configuration, le DGA devient un personnage charnière, responsable d'un pôle de services et, en même temps, en prise directe avec le politique, d'un côté la présidence (via le cabinet) dont il doit mettre en œuvre les projets et de l'autre, le ou les VP qui peu ou prou cherchent à faire valoir des propositions, potentiels vecteurs de leur notoriété. Le DGS souligne la démarche pédagogique qu'il revient au DGA de mener à bien, à l'endroit de vice-présidents qui manquent parfois d'expérience, notamment en matière de fonctionnement des services de la région et des administrations des autres partenaires publics et privés. La forte stabilité des postes de DGS et de directeur de cabinet en poste depuis 1998 et 2004 renforce ce pilotage étroit. Pour autant, vis-à-vis de la DGS, le rôle du cabinet est loin d'être négligeable : il constitue un " cordon de veille » technique et territoriale autour du président, assurant une protection vis-à-vis des erreurs de jugement de DGA, trop sûrs d'eux et capables, inconsciemment, de "rentrer dans un mur en klaxonnant " (Directeuradjoint de cabinet de la région $\mathrm{R} 4)$.

\section{Conclusion}

L'évolution d'ensemble de l'activité des régions au sein de l'action publique a favorisé la constitution d'une instance stratégique nouvelle, régulièrement étoffée, la direction générale des services. Des innovations statutaires, comme la création d'emplois fonctionnels relevant conjointement d'une forte technicité et d'un lien de confiance avec le chef de l'exécutif régional, ont renforcé la légitimité de cette instance ; mais cela s'est opéré au prix d'une tension pérenne, tant sont poreuses les responsabilités techniques et politiques (au point d'ailleurs que la distinction en devient parfois problématique, ce dont témoignent des crises ouvertes).

Il n'en reste pas moins que les modes de gouvernance, les configurations organisationnelles et les modalités d'accès à cette haute fonction publique régionale diffèrent

24. Dans la Région R1, l'organisation des services s'en trouve plus resserrée avec une équipe de confiance autour du DGS : "maintenant quil y a des DGA de valeur, il n'y pas grand-chose qui m'échappe " (DGS de la région $\mathrm{R} 1$ ).

25. Ainsi si les DGA quittent leur poste, ils ne sont pas réaffectés à une autre DGA; ils partent en retraite ou font une mobilité, soit dans le cadre d'une promotion (Inspection générale des Affaires sociales) ou pour prendre la DGS d'une structure intercommunale d'une autre région ou encore d'une mairie de la région. 
sensiblement. Dans les deux figures types identifiées (pilotage politique de l'institution régionale d'une part, et DGS " gouvernementale ", de l'autre), l'incertitude sur les frontières entre activité politique et pilotage administratif prévaut et expose les parties prenantes à l'instabilité de leurs positions respectives. Au-delà, elles sont portées par des marchés des places de direction générale très opposés ; l'un centré sur une régulation interne, l'autre sur le recours aux compétences externes, en priorité auprès des agents du partenaire clé des régions que reste l'État. Dépendance et proximité avec les élus dans un cas, légitimité reconnue et autonomie professionnelle de l'autre.

Ces deux configurations vont devoir affronter des contraintes inédites. L'environnement des collectivités territoriales est en pleine évolution : transfert de compétences, réforme fiscale, réagencement des rôles de l'État et des autres collectivités, attentes croissantes des usagers... autant de changements qui vont considérablement modifier le paysage territorial (Chabert, 2011). Ce contexte très mouvant aura probablement une forte incidence sur la gestion des collectivités.

Ces évolutions n'épargnent pas non plus la sphère politique : de nouveaux équilibres se sont instaurés au sein des majorités régionales élues en 2010 ; à terme, une nouvelle génération de responsables politiques de collectivités devrait naître avec le principe du mandat exécutif unique : plus présents dans et pour leur collectivité, ils seront sans doute plus tentés de s'affirmer aussi comme des chefs de services. D'ores et déjà, des évolutions se font jour. Le premier modèle tente de rationaliser et professionnaliser son mode de fonctionnement. Le second est conduit à refonder la relation entre les services et les élus ${ }^{26}$ sous l'emprise des groupes minoritaires de la majorité régionale. Dans quelle mesure le flou qui caractérise les rapports entre les responsables de l'exécutif et les hauts cadres de l'administration régionale sera-t-il une opportunité d'élaborer de nouveaux compromis raisonnables ou plutôt l'aiguillon de tensions croissantes ?

\section{Bibliographie}

Bachelet F. (2006), "Sociologie, formation et carrière des hauts fonctionnaires territoriaux 》, Annuaire des collectivités locales, Paris, CNRS Éditions.

Bel M. \& alii (2003), La décentralisation de la formation professionnelle : un processus en voie d'achèvement?, La Tour d'Aigues, Éditions de l'Aube.

Biland E. (2009), La fonction publique territoriale, Paris, La Documentation française, coll. "Problèmes politiques et sociaux », n 967.

26. Il s'agirait notamment de faire en sorte que les services rendent plus de comptes à l'ensemble de l'exécutif et moins exclusivement au président et qu'en outre, ils soient plus à l'écoute de la composante délibérative du conseil régional, notamment pour l'aider à mieux remplir sa fonction politique éminente, la discussion des textes qui lui sont soumis. 
Biland E. (2012), La fonction publique territoriale, Paris, La Découverte.

Buisson-Fenet H., Verdier E. (2011), «Une régionalisation impossible ? La régulation des formations professionnelles initiales en France : concurrences institutionnelles et hiérarchie d'instruments statistiques ", Sociologie et sociétés, vol. 43, n² 2, pp. 41-66.

Catlla M. (2007), Le travail public régional, Toulouse, Octarès.

Chabert J.-B. (2011), "Les régions face à leur avenir : "animation prospective” ou "gouvernement méso" ", Pouvoirs locaux, n 91 vol. IV, pp. 15-22.

Demazière D., Le Lidec P. (2008), «Introduction au dossier : la politique, un objet pour la sociologie du travail ", Sociologie du travail, 2, pp. 4-13.

Friedberg E. (1993), Le pouvoir et la règle. Dynamiques de l'action organisée, Pais, Seuil.

Jeannot G. (2005), "Les métiers flous du développement rural », Sociologie du travail, vol. $47, \mathrm{n}^{\circ} 1$, pp. 17-35.

Lanfranchi J. (1992), “Tournois et carrières », Travail et Emploi, $\mathrm{n}^{\circ}$ 54, pp. 72-85.

Le Lidec P. (2012), "Travail politique " in Bevort A., Jobert A., Lallement M., Mias A. (dir.), Dictionnaire du travail, Paris, PUF, Paris, pp. 804-809.

Mattina C. (2004), « Mutations des ressources clientélaires et construction des notabilités politiques à Marseille (1970-1990) », Politix, 67, pp. 129-155.

Nay O. (2002), "La négociation en régime d'incertitude : une comparaison des partenariats publics régionaux dans la mise en œuvre de la politique européenne ", Revue internationale de politique comparée, $\mathrm{n}^{\circ}$ 9, vol. 3, pp. 409-425.

Nay O., Smith A. (dir.) (2002), Le gouvernement du compromis, Courtiers et généralistes dans l'action publique, Paris, Economica.

Padioleau G. (1994), "Pour qui sonne le glas ? ", Préface à Bezes P., L'action publique volontariste, L'Harmattan, Paris.

Salais R. (1998), "Action publique et conventions : "état des lieux" " in Commaille J. et Jobert B. (Eds.), Les métamorphoses de la régulation politique, Paris, L.G.D.J., pp. 55-82. 